\title{
Influence of polarization of free-electron system in semiconductor on the position of minimum in plasma light reflection
}

\author{
V.S. Severin \\ National Aviation University, Chair of theoretical physics \\ 1, Cosmonaut Komarov prospect, 03058 Kyiv, Ukraine \\ E-mail: severinvs@ukr.net
}

\begin{abstract}
The dielectric permeability of the free-electron system in semiconductor is usually considered using the Drude-Lorentz model without taking into account this system polarization. But it seems reasonable to include polarization phenomena into consideration of the free-electron system behavior. In this paper, the position of minimum in plasma optical reflection by the system of free electrons is analyzed with allowance for this system polarization. This position can substantially differ from that given via calculation of it within the framework of the traditional Drude-Lorentz model. This difference is significant when analyzing the available experimental results.
\end{abstract}

Keywords: dielectric permeability, polarization, optical properties, reflection of light.

Manuscript received 06.05.10; accepted for publication 16.03.11; published online 30.06.11.

\section{Introduction}

Researching the plasma reflection of light by free electrons in semiconductor is one of the methods to determine electron effective mass $m$, concentration $n$ and the high-frequency dielectric permeability of crystal lattice $\varepsilon_{0}$ [1-6].

The light frequency value to produce the luminous reflectance minimum is a matter of principle in this method. The traditional Drude-Lorentz model is now utilized to find the above mentioned frequency [1-6].

Optical properties of substance are given by means of its dielectric permeability expressed in terms of this substance specific internal conductivity. However, the substance specific external conductivity is traditionally used in the dielectric permeability. It is the approximation not taking this substance polarization into account $[7,8]$.

The internal conductivity of a system of free electrons can considerably differ from the conductivity of the Drude-Lorentz model subject to the light frequency $[7,8]$. The Drude-Lorentz model does not consider the above polarization and can be applied only in the approximation of the large light frequency $\omega$, when the condition $\omega^{2}>>\omega_{p}^{2}$ is satisfied, where $\omega_{p}$ is the plasma frequency $[7,8]$.

The dielectric permeability of the free-electron system in semiconductor is considered taking the polarization of such a system into account and in the Drude-Lorentz model that does not consider this system polarization in the second section of this paper.

The location of the plasma minimum in luminous reflectance of the free-electron system is considered taking the polarization of this system into account and without this account in the third section. This location can substantially differ from the position given via calculation of it within the framework of the traditional Drude-Lorentz model. This difference is substantial when analyzing experimental results.

\section{Dielectric permeability of the Drude-Lorentz model. Inclusion of polarization into the dielectric permeability}

The Drude-Lorentz model examines free within an energy band electrons as an electronic gas neutralized by a positive crystal lattice background having the dielectric permeability $\varepsilon_{0}$ not depending on the light frequency. The motion equation of an electron being under action of the external electric field $\mathbf{D}(t)$ applied to a substance has the following look in this model:

$$
\frac{d \mathbf{v}(t)}{d t}=\frac{e}{m} \frac{\mathbf{D}(t)}{\varepsilon_{0}}-\frac{1}{\tau} \mathbf{v}(t) \text {. }
$$

Here, $t$ is time; $e$ is the electron charge; $\mathbf{v}(t)$ is its velocity; $\tau=1 / \gamma$ is the electron momentum relaxation 
time taking into account this electron scattering by crystal lattice.

The electric current of these electrons having the density $\mathbf{j}(t)=e n \mathbf{v}(t)$ arises under action of this field $\mathbf{D}(t)$. Here, $n$ is the electron concentration.

The external electric field $\mathbf{D}(t)=\mathbf{D}_{\omega} \exp (i \omega t)$ having the amplitude $\mathbf{D}_{\omega}$ creates the alternating current of these electrons with the amplitude of electric current density $\mathbf{j}_{\omega}$. The equation (1) implies the following form of the linear response of this current to the external field D:

$\mathbf{j}_{\omega}=s(\omega) \frac{1}{\varepsilon_{0}} \mathbf{D}_{\omega}$,

where

$$
s(\omega)=\frac{n e^{2} \tau}{m(1+i \omega \tau)}
$$

is the specific conductivity of free electrons within the Drude-Lorentz model. This conductivity is the external one in compliance with its calculation.

The following dielectric permeability is traditionally used to consider optical properties of free electrons [1-6]:

$\varepsilon_{S}(\omega)=\varepsilon_{0}+\frac{4 \pi}{i \omega} s(\omega)=\varepsilon_{0}\left(1-\frac{\omega_{p}^{2}}{\omega(\omega-i \gamma)}\right)$,

where

$\omega_{p}^{2}=\frac{4 \pi n e^{2}}{\varepsilon_{0} m}$

is the plasma frequency squared. This dielectric permeability is based on the Drude-Lorentz model.

However, the Drude-Lorentz model does not consider screening the external field $\mathbf{D}$ by mobile electrons of a substance. This screening begins to reveal itself at the time of the order of $t_{p}=\omega_{p}^{-1}$ [9]. Therefore, at the time of $t>t_{p}$ (that is at the light frequency of $\left.\omega<\omega_{p}\right)$ this substance conductivity electrons will be under action of not the external in relation to them field $\mathbf{D} / \varepsilon_{0}$, but they will experience action of the field $\mathbf{E} \neq \mathbf{D} / \varepsilon_{0}$, which is the internal field in the system of these electrons. The difference between the fields $\mathbf{E}$ and $\mathbf{D} / \varepsilon_{0}$ is caused by the vector of polarization $\mathbf{P}$ for the system of electrons [9-14]:

$$
\mathbf{P}=\left(\mathbf{D}-\varepsilon_{0} \mathbf{E}\right) /(4 \pi) \text {. }
$$

This difference is not considered when deriving the expression (3) for the dielectric permeability $\varepsilon_{S}(\omega)$.

Substance optical properties on the light frequency $\omega$ are defined by its dielectric permeability $\varepsilon(\omega)$. Derivation of the expression (3) for the substance dielectric permeability does not consider screening the external field by this substance charges that are optically active at the light frequency $\omega$ under study.
Indeed, the current density $\mathbf{j}$ can be expressed both through the internal electric field $\mathbf{E}$ and the field $\mathbf{D} / \varepsilon_{0}$ $[9,10,12-14]$

$\mathbf{j}_{\omega}=\sigma(\omega) \mathbf{E}_{\omega}=s(\omega) \mathbf{D}_{\omega} / \varepsilon_{0}$,

where $\mathbf{E}_{\omega}$ is the amplitude of internal electric field. The conductivity $s(\omega)$ determines the current response to the external field $\mathbf{D} / \varepsilon_{0}$ (with respect to substance charges being optically active at the light frequency $\omega$ under study), but the conductivity $\sigma(\omega)$ gives the current response to the internal field $\mathbf{E}$. Consequently, the physical quantity $s(\omega)$ is named as the substance external conductivity and the physical quantity $\sigma(\omega)$ is named as the internal one [12-14]. The distinction of the conductivity $\sigma(\omega)$ from the conductivity $s(\omega)$ in the expression (5) is caused by the difference of the polarization $\mathbf{P}$ (4) from zero.

Substance charges not partaking in optical transitions in the studied frequency interval create the frequency-independent background contribution $\varepsilon_{0}$ to this substance dielectric permeability $[1,11]$. Therefore, the expression for this substance dielectric permeability $\varepsilon(\omega)$ has the following form:

$\varepsilon(\omega)=\varepsilon_{0}+\frac{4 \pi}{i \omega} \sigma(\omega)$,

The expression (6) is derived from the relationship: $\mathbf{D}_{\omega}=\varepsilon(\omega) \mathbf{E}_{\omega}$,

which is the definition of substance dielectric permeability $\varepsilon(\omega)$, and from the relations (4), (5) [9-14]. The expression

$\mathbf{j}(t)=\frac{d}{d t} \mathbf{P}(t)$

is used under this derivation [9-14].

The definition of substance dielectric permeability requires the presence of this substance internal conductivity $\sigma(\omega)$ in it, but not the external one $s(\omega)$ [9-14]. For simplicity, the quantities $\sigma$ and $s$ are here supposed as scalar quantities, but not as tensor ones. The formulas (5), (7) give the next relationship:

$$
\sigma(\omega)=s(\omega) \varepsilon(\omega) \frac{1}{\varepsilon_{0}} .
$$
relation:

In addition, the formulas (6), (9) give the following

$$
\sigma(\omega)=\frac{1}{1-\frac{4 \pi}{i \omega \varepsilon_{0}} s(\omega)} s(\omega)
$$

After substituting the relation (10) into the formula (6) and taking the formula (2) into account, the next relationship is resulted: 


$$
\varepsilon(\omega)=\frac{\varepsilon_{0}}{1-\frac{4 \pi}{i \omega \varepsilon_{0}} s(\omega)}=\frac{\varepsilon_{0}}{1+\frac{\omega_{p}^{2}}{\omega(\omega-i \gamma)}} .
$$

The expression (11) for the dielectric permeability $\varepsilon(\omega)$ taking the above-stated polarization into account has a considerable difference from the approximate expression for the dielectric permeability $\varepsilon_{S}(\omega)(3)$, in which this polarization is not taken into account.

Having expanded the right side of the expression (11) into series by powers of $\left(\omega_{p} / \omega\right)^{2}$, we ascertain the validity of the following relationship:

$\varepsilon(\omega)=\varepsilon_{S}(\omega)+O\left(\left(\frac{\omega_{p}}{\omega}\right)^{4}\right)$

Therefore, the dielectric permeability $\varepsilon_{S}(\omega)$ not taking the polarization influence into account can be used only at the approximation of large frequencies, as soon as the condition $\omega^{2}>>\omega_{p}^{2}$ is satisfied.

The formula (3) gives the following expressions for real and imaginary parts of $\varepsilon_{S}(\omega)$ :

$$
\begin{aligned}
& \varepsilon_{S 1}(\omega)=\operatorname{Re}\left(\varepsilon_{S}(\omega)\right)=\varepsilon_{0}\left(1-\frac{\omega_{p}^{2}}{\gamma^{2}+\omega^{2}}\right), \\
& \varepsilon_{S 2}(\omega)=-\operatorname{Im}\left(\varepsilon_{S}(\omega)\right)=\varepsilon_{0} \frac{\omega_{p}^{2}}{\omega} \frac{\gamma}{\gamma^{2}+\omega^{2}} .
\end{aligned}
$$

However, the formula (11) gives the real part and the imaginary part of $\varepsilon(\omega)$ as follows:

$$
\begin{aligned}
& \varepsilon_{1}(\omega)=\operatorname{Re}(\varepsilon(\omega))= \\
& =\varepsilon_{0}\left[1-\frac{\omega_{p}^{2}\left(1+\left(\omega_{p} / \omega\right)^{2}\right)}{\gamma^{2}+\omega^{2}\left(1+\left(\omega_{p} / \omega\right)^{2}\right)^{2}}\right], \\
& \varepsilon_{2}(\omega)=-\operatorname{Im}(\varepsilon(\omega))= \\
& =\varepsilon_{0} \frac{\omega_{p}^{2}}{\omega} \frac{\gamma}{\gamma^{2}+\omega^{2}\left(1+\left(\omega_{p} / \omega\right)^{2}\right)^{2}} .
\end{aligned}
$$

\section{Minimum of plasma optical reflection}

The reflectivity of a body for the normal light incidence $R(\omega)$ is given by the next expression $[1,3-6]$ :

$$
R(\omega)=\frac{(N(\omega)-1)^{2}+K(\omega)^{2}}{(N(\omega)+1)^{2}+K(\omega)^{2}}
$$

Here,

$$
K(\omega)=\sqrt{\frac{1}{2}\left(-\varepsilon_{1}(\omega)+\sqrt{\varepsilon_{1}^{2}(\omega)+\varepsilon_{2}^{2}(\omega)}\right)}
$$

is the absorption index of body substance;

$$
N(\omega)=\sqrt{\frac{1}{2}\left(\varepsilon_{1}(\omega)+\sqrt{\varepsilon_{1}^{2}(\omega)+\varepsilon_{2}^{2}(\omega)}\right)}
$$

is the refraction index of it.

Under the assumption of weak light absorption, when the condition $N^{2}(\omega)>>K^{2}(\omega)$ (or $\left.\varepsilon_{1}^{2}(\omega)>>\varepsilon_{2}^{2}(\omega)\right)$ is satisfied, the formula (17) gives

$R(\omega)=\left(\frac{N(\omega)-1}{N(\omega)+1}\right)^{2}$.

It is already here $N(\omega)=\sqrt{\varepsilon_{1}(\omega)}$. In this case, the minimum value of reflectivity $R(\omega)=0$ occurs under the following condition:

$\varepsilon_{1}(\omega)=1$.

The weak light absorption by a system of free electrons takes place at the fulfillment of the condition $\omega^{2}>>\gamma^{2}$. The substitution of dielectric permeability $\varepsilon_{S 1}(\omega)$ derived from the formula (13) at the approximation $\gamma=0$ into the equation (21) gives the reflectivity minimum at the following frequency $[1$, $5,6]$ :

$\omega_{1}=\omega_{p} \sqrt{\frac{\varepsilon_{0}}{\varepsilon_{0}-1}}$.

This frequency value that is derived using the Drude-Lorentz model to obtain the dielectric permeability $\varepsilon_{S}(\omega)$ does not consider the influence of polarization.

The substitution of the dielectric permeability (15) $\varepsilon_{1}(\omega)$ into the equation (21) (at the approximation $\gamma=0$ ), under which derivation the influence of polarization is taken into account, gives the reflectivity minimum at the following frequency:

$\omega_{2}=\omega_{p} \frac{1}{\sqrt{\varepsilon_{0}-1}}$.

It follows from the formulas (22), (23) that there is $\omega_{2}=\omega_{1} \frac{1}{\sqrt{\varepsilon_{0}}}$.

\section{Discussion}

The electric field in a substance influencing on charge carriers differs from the external one because of this substance polarization.

The available methods to account the substance polarization: Clausius-Mossotti, Lorentz-Lorenz, Ewald, Onsager $[2,3,10,15]$ examine the difference between the electric field in a substance, which influences on atoms (molecules) of this substance, and an external electric field as if this difference is caused by the substance polarization at the location of atoms (molecules). 
These methods, in essence, utilize models of the spatial localization of electric charges and are unsuitable for mobile charges delocalized in space. Electrons being free within the energy band of a solid are exactly such charges. The Drude-Lorentz model that is traditionally used to study optical properties of these electrons does not take the influence of substance polarization into account and can be applied in the approximation of only high frequencies $\omega^{2}>>\omega_{p}^{2}$.

The examination method of the influence of substance polarization used in this paper is applicable to free electrons and allows using the results of the traditional Drude-Lorentz model. The position of minimum of plasma optical reflection obtained within the framework of this method substantially differs from the position of this minimum obtained within the framework of the traditional Drude-Lorentz model. For example, it is $\varepsilon_{0}=16$ for germanium $[3,6]$ and the formula (24) gives $\omega_{1}=4 \omega_{2}$ in that case.

This disagreement must be taken into account when determining the free electron effective mass, concentration and the high-frequency dielectric permeability of crystal lattice by means of reflection spectra for free electrons.

Experiment gives the minimum in optical reflection of free electrons at the wavelength $\lambda_{1}=5.7 \mu \mathrm{m}$ for the case of $n$-Ge having the electron concentration $8.09 \cdot 10^{19} \mathrm{~cm}^{-3}$ (p. $\left.249[6]\right)$.

The formula (22) no considering the influence of substance polarization gives the plasma frequency (which is designated in this case as $\omega_{p s}$ )

$\omega_{p s}=\omega_{1} \sqrt{\frac{\varepsilon_{0}-1}{\varepsilon_{0}}}=\frac{2 \pi c}{\lambda_{1}} \sqrt{\frac{\varepsilon_{0}-1}{\varepsilon_{0}}}$,

where $c$ is the velocity of light. The expression (25) gives $\omega_{p s}=3.2 \cdot 10^{15} \mathrm{~s}^{-1}$ under $\varepsilon_{0}=16$.

The formula (23) considering the influence of substance polarization gives the plasma frequency (which is designated in this case as $\omega_{p}$ )

$\omega_{p}=\omega_{2} \sqrt{\varepsilon_{0}-1}=\frac{2 \pi c}{\lambda_{1}} \sqrt{\varepsilon_{0}-1}$.

The expression (26) gives $\omega_{p}=1.28 \cdot 10^{16} \mathrm{~s}^{-1}$ under $\varepsilon_{0}=16$. Evidently that there is the substantial difference between values $\omega_{p}$ and $\omega_{p s}$.

The electron effective mass determines the plasma frequency value. Having designated

$\omega_{p s}^{2}=\frac{4 \pi n e^{2}}{\varepsilon_{0} m_{s}}, \omega_{p}^{2}=\frac{4 \pi n e^{2}}{\varepsilon_{0} m}$,

we obtain the next relationship between effective masses

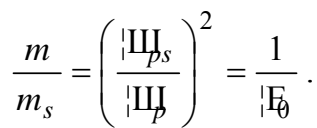

Here $m_{s}$ is the electron effective mass compared with the plasma frequency $\omega_{p s} ; m$ is the electron effective mass compared with the plasma frequency $\omega_{p}$.

The formula (27) gives the value $\mathrm{m} / \mathrm{m}_{\mathrm{s}}=0.06$ for the case of $\varepsilon_{0}=16$. Results having a difference up to $60 \%$ were obtained under the experimental determination of the electron effective mass for Ge by means of reflection spectra (p. 250-251 [6]). These results differ from values obtained by means of electric measurements by $25 \%$ (p. 251 [6]). Moreover, the comparison of them with results obtained from the cyclotron resonance gives a difference more than twice as much (p. 253 [6]). Possibly, such distinctions are the display of the polarization influence not taken into account when treating the experimental results.

\section{References}

1. Yu. Madelung, Theory of Solids. Nauka, Moscow, 1980 (in Russian).

2. J.C. Slater, Insulators, Semiconductors and Metals. Mir, Moscow, 1969 (in Russian).

3. P. Grosse, Free Electrons in Solids. Mir, Moscow, 1982 (in Russian).

4. K. Seeger, Semiconductor Physics. Mir, Moscow, 1977 (in Russian).

5. I.M. Tsidil'kovskiy, Band Structure of Semiconductors. Nauka, Moscow, 1978 (in Russian).

6. Yu.I. Ukhanov, Optical Properties of Semiconductors. Nauka, Moscow, 1977 (in Russian).

7. O.V. Vakulenko, V.S. Severin, Reflection of light by metal with account of polarization of conductance electrons // Metallofizika i noveishie tekhnologii 29 (1), p. 41-55 (2007), in Russian.

8. O.V. Vakulenko, V.S. Severin, Calculation of the metal reflectivity with taking polarization into consideration // Semiconductor Physics, Quantum Electronics \& Optoelectronics 10 (1), p. 55-59 (2007).

9. F. Platzman, P. Volf, Waves and Interactions in Plasma of Solids. Mir, Moscow, 1975 (in Russian).

10. Yu.A. Il'inskiy, L.V. Keldysh, Interaction of Electromagnetic Radiation with Matter. Tutorial. Moscow State University Publ., Moscow, 1989 (in Russian).

11. S.I. Pekar, Optics of Crystals and Additional Light Waves. Naukova dumka, Kiev, 1982 (in Russian).

12. R. Kubo, The fluctuation-dissipation theorem // Repts. Progr. Phys. 29, Pt. 1, p. 255-284 (1966).

13. T. Izuyma, An expansion theorem for the electric conductivity of metals. I. Electric conductivity for longitudinal electric field // Progr. Theor. Phys. 25 (6), p. 964-980 (1961).

14. R. Eykholt, Extension of the Kubo formula for the electric-conductivity tensor to arbitrary polarization of electric field // Phys. Rev. B 34 (10), p. 66696674 (1986).

15. N.G. Bakhshiev, Introduction to Molecular Spectroscopy. Published in Leningrad University, Leningrad, 1987 (in Russian). 